\title{
La coherencia en lexicografía. \\ El caso de algunos indoamericanismos en el Diccionario de Autoridades (1726-1739)
}

Rafael Cala Carvajal

Universitat de Barcelona

\section{OBJETIVOS}

Impregnada la mentalidad de los europeos de fantasías y leyendas, los relatores de Indias idealizaron América y proyectaron su yo sobre el ellos identificando y comparando lo nuevo con lo conocido (vid. Borrero Barrera 2000-2001), proceder trasladado a los diccionarios setecentistas ${ }^{1}$. En este sentido, nuestra intención es analizar el tratamiento lexicográfico de los indigenismos caimán, iguana, chinchilla, guacamayo y manato (manatî) ${ }^{2}$ en el Diccionario de Autoridades (DA) con miras a constatar, primero, el carácter enciclopédico de las definiciones de los susodichos indoamericanismos; $y$, segundo, si este favorece que el tratamiento lexicográfico de los préstamos indígenas sea coherente ${ }^{3}$.

1 El paralelismo trazado entre la fauna americana y la europea se lleva a cabo en el Diccionario de Autoridades, como en las crónicas de Indias, con el símil ("tamaños y mayores que palomas", "otros hai tan pequeños como xilgueros", s. v. papagayo; "Ave algo mayór que el papagáyo", s. v. guacamayo; y "Animaléjo pequeño como hardilla", s. v. chinchilla); con los grupos sintagmáticos "à modo de" (s. v. caiman, arda) y "à manera de" (s. v. iguana); y, en último término, con el adjetivo "semejante" (s. v. caiman).

2 El polimorfismo en que están los vocablos manato y manatí en el s. XVIII ("Otros dicen en Cast. Manati”, DCCA, s. v. manato) tiene que ver con su etimología científica (DCEC, s. v. manatí) y popular ("por razon de tener las manos delanteras le llamaron Manato", DCCA, s. v. manato).

3 Para ello aplicamos las palabras de Weinreich (1970: 70), quien apuntó que "la cohe- 
Esto es, nos preguntamos, por un lado, si el DA, después de identificar un animal americano con otro europeo, retoma dicha identificación en este último $\mathrm{y}$, por el otro lado, en el supuesto de que el DA no la retomara, si ello se debe al enciclopedismo de las entradas.

\section{DEFINICIÓN LEXICOGRÁFICA VS. DEFINICIÓN \\ ENCICLOPÉDICA EN EL DICCIONARIO DE AUTORIDADES}

Como toda lengua evoluciona y se acomoda a las necesidades comunicativas de sus hablantes, el español tuvo que satisfacer en América unas necesidades lingüísticas desconocidas en España, cosa que contribuyó a la gestación de la singularidad lingüística americana del castellano, ya consolidada a comienzos del siglo XVII (vid. Guitarte 1984) si bien hunde sus raíces en las postrimerías del siglo XV.

En efecto, el primer lexicógrafo español en lematizar un indigenismo (canoa) fue el andaluz Elio Antonio de Nebrija, lo cual no debe extrañarnos habida cuenta de que a Andalucía arribaban los galeones de las Indias (vid. Frago Gracia 1994: 139-185). Con todo, es fundamentalmente a partir del siglo XVII cuando encontramos indoamericanismos en los diccionarios monolingües y bilingües (vid. Lope Blanch 1977; Gútemberg 1984) ${ }^{4}$, tendencia heredada por el DA (vid. Lázaro Carreter 1980: 88-94; Salvador Rosa 1985; Romero 1992; Alvar Ezquerra 1993b; y Azorín Fernández 2000: 159-199), aun cuando no menciona América a diferencia de Esteban de Terreros y Pando, cuyo conocimiento sobre la realidad americana contó con los excelentes trabajos de sus hermanos de congregación (vid. Gutiérrez Cuadrado y Pascual 1996):

rence de la lexicographie pourrait être améliorée si les auteurs de dictionnaires adhéraient à l'hypothèse selon laquelle les mots d'une langue sont [...] complementaires [...] là où la signification d'un terme finit, celle d'un autre commence".

4 Durante el s. XVIII ven la luz diccionarios del español de América como el Diccionario de voces americanas (1751-1777) de Manuel José de Ayala; el Vocabulario de las voces provinciales de América, en el Diccionario geográfico-histórico de las Indias Occidentales o América (1786-1789), de Antonio de Alcedo; y el Diccionario provincial de la Isla de Cuba (1795), de Peñalver.

5 Salvando las distancias, el DA respondería a lo que en la lexicografía moderna se de- 
En el cuerpo de esta obra [...] se ponen várias voces peculiares y própias, que se usan freqüentemente en algunas províncias y réinos de España, como en Aragón, Andalucía, Astúrias, Murcia, \&c. [...] (DA, Prólogo, V); y

No es menester pasar a las Indias, en las cuales, omitidos sus innumerables idiomas nativos [...], dan casi en cada reino y provincia diverso nombre a un mismo objeto [...] (DCCA 1987: IV).

Por nuestra parte, en el presente artículo obviamos la problemática terminológica sobre los conceptos de americanismo e indigenismo (indoamericanismo, indigenismo americano...) (vid. Rabanales 1963; Rona 1969; Gútemberg 1984; Martinell Gifre 1992; Fontanella de Weinberg 1992; Buesa y Enguita 1992; Pottier-Navarro 1992; y Galeote 1997), de suerte que nos centraremos en la consideración teórica que gira en torno al diccionario y la enciclopedia.

Si harto complejo es el concepto de definición, no lo es menos el de diccionario y el de enciclopedia (vid. Haiman 1980, 1982; Frawley 1981; Lara 1989; Ahumada Lara 1989; Anglada Arboix 1991; Alvar Ezquerra 1993a; Gutiérrez Cuadrado 1996; y Haensch 1999); de manera que nos ceñimos a la definición que Fernández Sevilla propone de diccionario en tanto que nos permitirá contrastarlo con la enciclopedia:

el diccionario es un conjunto de signos pertenecientes a la lengua; por tanto, da cabida a todas las palabras y [a] todas sus clases, con excepción de los nombres propios. [...] La enciclopedia, por el contrario, reúne información sobre $[. .$.$] cosas existentes en una civiliza-$ ción [...] (1974: 66).

En estrecha dependencia de lo anterior, los parámetros teóricos en que descansa la tipología de la definición en la lexicografía son, grosso modo, dos. En primer lugar, tenemos el del metalenguaje empleado en la microestructura; en segundo lugar, el de la naturaleza de lo definido y la información proporcionada en la parte definitoria. Según el primero, la definición es propia si el lexicógrafo emplea la metalengua de contenido; e impropia, si recurre a la metalengua de signo. De acuerdo con el segundo, es enciclopédica (real, o lógica) o lexicográfica (nomi- 
nal): aquella debe identificar con claridad el objeto definido, mientras que esta ha de enumerar los rasgos semánticos suficientes para deslindar la palabra definida del resto de unidades léxicas (vid. Rey-Debove 1971: 180-257; Bosque 1982; Werner 1982; Seco 1987a: 32; y Casares 1992: 158-161).

En relación con la idea de 'suficiencia', tenemos que "la definición, para ser tal, es teóricamente una información sobre todo el contenido y nada más que el contenido de la palabra definida" (Seco 1987a: 20), en razón de que Seco discrimina "los elementos constitutivos del significado [el contenido] y los elementos habituales del contexto [el contorno]" (1987b: 45). Consecuentemente, la concisión y la suficiencia en el contenido de la palabra definida desempeñan un papel destacado en la delimitación del diccionario y la enciclopedia.

De aplicar en el DA la definición de diccionario de Fernández Sevilla (vid. supra) y el criterio de la naturaleza de lo definido y la información proporcionada en la parte definitoria, el DA sería concebido más bien como un diccionario de lengua que como una enciclopedia ${ }^{5}$ porque, en primera instancia, diferencia entre el vocabulario y el diccionario:

[...] [la RAE] no le llama Vocabulario, porque en la Léngua Españóla se entienden [...] por Vocabularios los libros en que se expressan las voces, sin explicarlas, ni adornarlas con etymologías y phrases que se vuelven en otra Léngua [...] y por Diccionarios se entienden los libros, donde no solo se vierten en otra Léngua los vocablos, sino que se explica su naturaleza, y el sentído de las phrases [...] (DA, Prólogo, II);

en segunda instancia, porque no lematiza antropónimos y topónimos (DA, Prólogo, VI $)^{6}$ y, por último, porque persigue presentar con propiedad el significado de la entrada:

nomina diccionario enciclopédico, que Martínez de Sousa (1995) define como "diccionario en el que se mezclan un diccionario de lengua con descripción lingüística y una enciclopedia con descripción enciclopédica." (s. v. diccionario enciclopédi$\mathrm{co})$.

- 6 A pesar de que no hay unanimidad entre los lexicógrafos a la hora de lematizar nombres propios en los diccionarios generales de lengua -en la enciclopedia sí la hay-, es 
El amor à las letras, y la cultúra y pulidéz del trato humano ha reducido casi todas las Ciéncias, ò Artes à Diccionarios [...] pero la opinión de los Sabios es que la edición de los Diccionarios ha perjudicado mucho à la República literária, porque no se estudian las Ciéncias con sólidos fundamentos, sino por la ligéra superficie de la explicación de las voces, ò términos sueltos y divididos por Abecedario, en los Diccionarios. Esta evidente senténcia no se debe entender, ni comprehende à los Diccionarios de las Lénguas, donde se expressan los significados de las voces, su variédad, el sentído de cada una, sus phrases y elegáncia [...] y el méthodo mas regular y facil es el de los Diccionarios [...] y faltandole à la Léngua Españóla el suyo, ha sido este el principal empeño de la Académica, sin que sea su fin emendar, ni corregir la Léngua [...] sí solo explicar las voces, phrases, y locuciones, desterrar y dár à conocer los abusos introducidos [...] (DA, Prólogo, IV).

No obstante, el DA acepta en los lemas que analizamos notas enciclopédicas marginales al contenido del vocablo en razón de que la descripción era uno de los muchos procedimientos con que se incorporaba y explicaba la realidad americana en las crónicas de Indias, lo que propició que se atesorara en ellas un saber enciclopédico que incluía, por ejemplo, el provecho medicinal de la flora y la fauna americanas (vid. Gutiérrez Rodilla 1997-1998; y Borrero Barrera y Cala Carvajal, en prensa).

Finalmente, para concluir este apartado, anotamos que la mezcla de descripciones lingüísticas y enciclopédicas en el DA es herencia de la lexicografía medieval (vid. Buridant 1986; y Alvar Ezquerra 1995), que no deslindó los diccionarios ni de la gramática ni de la enciclopedia ${ }^{7}$,

práctica habitual omitirlos, a excepción de los derivados (franciscano, gongorino...) (vid. Clarinval 1967; y Lecomte Hilmy 1989).

7 Valga como muestra el siguiente testimonio de Isidoro de Sevilla:

4. [...] Aristóteles y Tulio consideraron que una definición completa de esta ciencia [...] tiene que partir del género y de las diferencias. 5. Algunos autores posteriores, ampliando esto, dividieron la perfecta definición sustancial en cinco partes, que vienen a ser como sus miembros: la primera, se refiere al género; la segunda, a la especie; la tercera, a la diferencia; la cuarta, a lo que le es característico; y la quinta, a lo que le es accidental (libro II, 4-5, p. 399).

8 Este se plasma en el caimán ("unas glándulas como habas [...] de que usan los In- 
situación que permaneció inalterada -sin desaparecer totalmente en función de la tradición lexicográfica- hasta el Siglo de las Luces, centuria durante la cual la eclosión de la ciencia moderna fragmentó el mundo enciclopédico tradicional.

En consecuencia, la diferencia entre el diccionario y la enciclopedia se ha construido histórica y culturalmente (vid. Gutiérrez Cuadrado 1996: 154), de modo que la labor lexicográfica de la RAE en el Diccionario de Autoridades sigue unos patrones heredados de los siglos precedentes (Sebastián de Covarrubias, los diccionarios académicos del italiano y del francés...) y de las fuentes -literarias y no literarias- que le suministraron las autoridades.

\section{LA COHERENCIA EN LA DEFINICIÓN DE CAIMÁN, IGUANA, CHINCHILLA, GUACAMAYO Y MANATO EN EL DICCIONARIO DE AUTORIDADES}

\subsection{CAIMÁN E IGUANA}

La lectura atenta de las definiciones del caimán y de la iguana (animales comparados con el lagarto y el cocodrilo) evidencia que la similitud difiere en cada una de ellas: el caimán y el cocodrilo se emparentan en el hábitat -el DA circunscribe el primero a América y el segundo, a África y América-, en la dureza de la piel y en su dentadura. Sin embargo, el lagarto apenas se asemeja a aquellos:

Insecto de colór verde, cuya piel áspera le hace parecer manchado. Tiene la cabeza grande y la cola larga, y quatro bracillos de la misma hechúra que los del hombre, aunque mui cortos. Anda como arrastrando; pero no por esso dexa de ser velocissimo, assi corriendo por tierra, como trepando por los árboles, paredes y bardáles. Habita en los huecos de los árboles, y lo mas ordinario en cuevecitas, en las bocas donde hai conéjos, de donde sale rara vez, sino es en dia de Sol [...] (s. v. lagarto).

Asimismo, la información contenida en la microestructura del caimán y del cocodrilo difiere en que la RAE menciona en el primero su 
aprovechamiento medicinal ${ }^{8}$ y su reproducción ovípara ("Pone los huevos en la aréna", s. v. caiman); en crocodrilo, en cambio, hace hincapié en que no usa la lengua ("Entre todos los animales de la tierra es el único que carece del uso de la lengua"), en la movilidad de su mandíbula superior ("tiene la singularidad de imprimir la mordedura con la mexilla superiór, que es movible") y en su sentido de la vista ("En tierra es mui perspicaz su vista; pero en el agua esta ciego").

Todo ello contrasta con la parquedad de la parte definitoria de la iguana, cuyo punto de unión con el lagarto descansa en el término científico Lacertus (Americanus):

[...] Es animal venenoso, y muerde con tanta fuerza, que dexa señalados los dientes en el hierro. Sale del Latino Lacertus, que significa lo mismo. (s. v. lagarto); y

Animal amphibio de la America, el qual es à manera de un lagarto, y de quien se trahen a España unas piedras llamadas de Iguana, que son provechosas para algunos males. Lat. Lacertus Americanus [...] (s. v. iguana);

y en la valoración negativa de los hablantes plasmada en las autoridades aducidas por la RAE en los respectivos lemas:

ACOST. Hist.Ind.lib.4.cap.38. Harto mejor comida es la de Iguánas; aunque su vista es bien asquerosa, pues parecen puros lagartos de España (s. v. iguana); y

Herr.Hist.Ind.Decad.I lib.8 cap.3. Padecieron tanta hambre, que ni sapos, ni ranas, ni lagartos, ni otras cosas vivas, por sucias, dexavan de comer (s. v. lagarto).

\subsection{CHINCHILLA}

En el caso de la chinchilla, el DA la compara con la ardilla (arda) sin otro rasgo en común que el tamaño ${ }^{9}$. Así y todo, mientras refiere en

dios", DA, s. v. caiman) y en la iguana ("se trahen a España unas piedras [...] provechosas para algunos males", DA, s. v. iguana), a diferencia del cocodrilo y del lagarto.

9 De igual manera procede Terreros y Pando en su diccionario: 
chinchilla cuán apreciada era la piel del roedor americano, en arda describe al animal, su hábitat y su alimentación:

Animaléjo pequeño como hardilla, que se cria en la sierra del Perú. Tiene el pelo mui blando, y sus pieles se trahen por cosa regalada y saludable para abrigar el estómago, y partes que tienen necessidad de calór moderado [...] (s. v. chinchilla); y

Animaléjo conocído à modo de una rata grande, o fuína pequeña. Tiene la cola mui grande y poblada de pelo: su colór es rúbio, y el pecho blanco. Criase en los montes donde hai pinos, y se mantiene de los piñones [...] (s. v. arda).

La mención de la "rata grande" y la "fuína pequeña" en el artículo arda ("Animaléjo conocído à modo de una rata grande, o fuína pequeña") no debe inducirnos a creer que se trate de animales emparentados habida cuenta de que se oponen en el hábitat, en la alimentación y en su concepción en el seno de la sociedad:

Se llama tambien cierta especie de ratón mucho mayór que el comun y doméstico, de colór obscuro, aunque tambien suelen hallarse blancas. Crianse regularmente en los molinos y casas de campo, y suelen crecer tanto que resisten y se defienden de los gatos. Matanlas, poniendolas una luz delante de los ojos, con la qual las encandilan y no se mueven [...] (s. v. rata); y

Especie de marta ò raposa del tamaño de un gato, de colór rubio que tira algo à negro, y por la barriga blanca. Es mui dañina y destruidóra de los gallinéros y palomáres [...] (s. v. fuína).

En nuestra opinión, la alusión a la "rata grande" y a la "fuína pequeña" (s. v. $a r d a$ ) cobra sentido gracias a la idea de tamaño aportada por los adjetivos grande y pequeña en tanto que la rata es "cierta especie de ratón mucho mayór que el común y el doméstico"; y la fuína, "especie de marta ò rapósa del tamaño de un gato". Por consiguiente, la ardilla es mayor que la rata, pero menor que la 'fuína' (comadreja).

Animál del Perú, de la magnitúd de la hardilla: su pelo es blando, y la piel mui estimada para el abrigo. [...] (DCCA, s. v. chinchilla); y

Animal pequeño, silvestre, mui lijero, y que salta en los arboles de rama en rama, valiendose para esto, como de cierta especie de ala, de una cola que tiene mui grande. [...] (DCCA, s. v. ardilla). 


\subsection{GuACAMAYO}

En cuanto al tratamiento lexicográfico del guacamayo y el papagayo, el DA no es coherente. En efecto, en papagayo se paralelan un animal conocido (el papagayo) y otro desconocido (el guacamayo): "se hallaron papagáyos distintos de los hasta entonces conocidos" (s. v. papagayo). Ahora bien, las especies americanas equiparadas al papagayo tendrían que figurar en guacamayo y no en papagayo:

Despues de descubiertas las Indias se hallaron papagáyos distintos de los hasta entonces conocidos, unos verdes, tamaños y mayores que palomas, que tienen un flueco de plumas blancas en el nacimiento del pico, otros que le tienen colorado como el carmesí. Hai otros de colas largas, y mayores de cuerpo, y tienen los encuentros de las alas colorados. Otros hai tan pequeños como xilgueros, todos verdes con mas de cien diferencias de plumas [...] (s. v. papagayo); y

Ave algo mayór que el papagáyo, y de su figúra. Tiene la pluma de varias y hermosas colores, y las de la cola mui largas. Son torpes para hablar, y jamás llegan à formar voces. Algunos los llaman Guacamáyas. Lat. Pseudopsithacus [...] (s. v. guacamayo).

Pese a esta inadecuación en la microestructura de papagayo ${ }^{10}$, no cabe duda de que el DA relaciona las dos aves en virtud de que comparten el atributo de imitar el lenguaje articulado humano ("torpes para hablar, y jamás llegan à formar voces" (DA, s. v. guacamayo); e "imita la voz del hombre y finge con propiédad su risa" (DA, s. v. papaga-

10 Terreros y Pando aporta la información en el lema esperable (guacamayo):

Ave parlera, verde, y de otros colores [...] Las especies que hai de papagayos son mas de veinte, y los mas hermosos no exceden la magnitud de un gorrion: el canto de estos semeja mucho al del pardillo: son verdes, y azules, $\mathrm{y}$ tan apacibles, que en menos de ocho dias se domestican [...] (DCCA, s. v. papagayo); y

Ave de hermosos colores, parecida al papagayo. [...] es de la magnitud de un capon, aunque mas largo, el pico negro, y encorvado en medio circulo; los ojos blancos, y negros, y en la parte inferior tres rayitas, que forman una $\mathrm{S}$, la parte superior de la cabeza lisa, y verde, la garganta con una especie de collar, el pecho, vientre, muslos, y parte posterior, é inferior amarillo; lo alto de la cabeza, la espalda, alas, y parte inferior de la cola de un hermoso azul; las piernas cortas, y las uñas mui corvas [...] (DCCA, s. v. guacamayo). 
yo)). Otro hecho que confirma su vinculación es la designación latina del guacamayo ("Pseudopsithacus") y del papagayo ("Psittacus").

\subsection{ManATO (MANATí)}

En manato, el DA identifica el manatí con el tiburón ("Especie de Tiburón, que se cria en el mar Indico", s. v. manato), pero ni la definición de uno ni la del otro avalan esta relación porque solo comparten la alusión a la longitud y al peso del animal y difieren en el modus de presentar la información ("el cuerpo mui gruesso, y del largo de veinte piés", s. v. manato; y "de monstruosa grandeza, hasta veinte pies de largo", s. v. tiburon). De igual manera, resalta el detallismo de la descripción del manatí -resultado del enciclopedismo del DA- frente a la concisión del artículo tiburon:

Especie de Tiburón, que se cria en el mar Indico, el qual tiene la boca como buey y el cuerpo mui gruesso, y del largo de veinte piés, cubierto de una piel duríssima è impenetrable. El lomo es llano, los ojos pequeños, y el colór pardillo. Tiene dos pies, que le salen como brazos de los hombros, de los quales se sirve para nadar. La hembra tiene dos grandes tetas, con cuya leche cria sus hijos, los quales pare vivos, como animal de tierra. Su carne, siendo fresca, tiene sabór de ternéra, y saláda tiene gusto de atún, aunque es mejór, y se conserva más tiempo. Su mantéca es mui buena, y nunca se enrancia, y con ella se adoba su mismo cuero, para hacer dél calzado y otras cosas. En la cabeza de este pescado dicen se cria una piedra mui provechosa contra las piedras de los riñones, y para los dolores de ijada [...] (s. v. manato); y

Pez marino, especie de perro, ù lobo; pero de monstruosa grandeza, hasta veinte pies de largo, y à proporcion gruesso: tiene solamente en todo el cuerpo la esquena, ò espinazo: es voracíssimo de carne humana [...] (s. v. tiburon).

En lo concerniente a las remisiones a otros animales, el manatí se compara con el buey por su parecido en la boca ("tiene la boca como buey", s. v. manato). Empero, el DA no aclara si se trata del buey (< BOS, BOVIS) o del buey marino, con el que el manatí comparte el término científico (Vitulus marinus): 
En la cabeza de este pescado dicen se cria una piedra mui provechosa contra las piedras de los riñones [...]. Lat. Vitulus marinus. (s. v. manato); y

Mónstruo que se cria en el mar,que por tener alguna semejanza con el buey [...] se llamó assi. Lat. Phoca, a. Vitulus marinus [...] (s. v. buey marino $)^{11}$.

Cuestión paralela a la mentada en el caso de buey para manato es la referencia al perro y al lobo en tiburon ("Pez marino, especie de perro, ù lobo", s. v. tiburon), debido a que el término latino del buey marino (vid. supra) y el del lobo marino son el mismo:

Pescado mui semejante en tamaño y figúra al Lobo terrestre, de quien se diferencia en que la piél es de colór mas claro, y tiene el pelo mui suave y brillante. [...] Lat. Vitulus marinus [...] (DA, s. v. lobo marino).

Para finalizar, el DA tampoco justifica la referencia en tiburon a perro, a no ser que partamos del parentesco entre este y el lobo ("una especie de perro sylvestre mui pernicioso y dañíno para los ganados", DA, s. v. lobo).

\section{CONCLUSIÓN}

Una vez argumentado que el DA es más un diccionario de lengua que una enciclopedia, a lo largo de nuestro trabajo nos hemos planteado si la RAE es coherente o no en el tratamiento lexicográfico de las voces americanas caimán, iguana, chinchilla, guacamayo y manato en el Diccionario de Autoridades, y si la coherencia o incoherencia resulta del enciclopedismo de las entradas ${ }^{12}$. Esto es, nos hemos cuestionado si el DA, tras identificar un animal americano con otro europeo, reto-

11 Terreros y Pando mantiene la referencia a la vaca y al buey marino ("Especie de Vaca, Becerro ó Buey marino"), si bien cuestiona la remisión al lobo ("Lebr. lo toma tambien mal por Lobo marino.") (DCCA, s. v. manato).

12 El DA ha sido objeto de estudio desde otros muchos puntos de vista, que no enumeramos aquí (vid. Cotarelo y Mori 1914; Pérez Goyena 1922; Gili Gaya 1963; Val 1992; Gutiérrez Rodilla 1993, 1994-1995 y Aliaga Jiménez 1994). 
ma dicha identificación en este último y, en el caso de no proceder así, si ello depende de su enciclopedismo ${ }^{13}$.

A este propósito, el DA es coherente parcialmente en caiman y en iguana, puesto que en crocodrilo sugiere implícitamente la identificación entre el cocodrilo y el caimán respecto al hábitat ("Criase en el rio Nilo y en otros de la América", s. v. crocodrilo; y "se cría en las rias de las Indias y en algunas Islas", s. v. caiman). Pero no lo es al relacionar el lagarto con el caimán porque el lagarto no se hace eco de ello; al contrario, emparentar el lagarto y la iguana sí es pertinente, y sus definiciones no lo contradicen ni en el étimo (Lacertus vs. Lacertus americanus) ni en la estimación social peyorativa del referente ${ }^{14}$.

En el resto de indigenismos, la coherencia varía: entre chinchilla y arda el vínculo es mínimo (el tamaño); en papagayo y guacamayo, la incoherencia radica en que la información sobre el 'papagayo americano' no se ofrece en la entrada esperable (guacamayo). Finalmente, en manato y tiburon hallamos el mayor grado de incoherencia en razón de que el manato no es un tiburón, ni viceversa. Sin duda, la ambigüedad con que el manatí se refiere al buey, y el tiburón al lobo, agrava la incoherencia, máxime si reparamos en que el manatí, el buey y el lobo marinos se designan en latín Vitulus marinus.

13 El caimán se identifica con el cocodrilo y el lagarto; la iguana, con el lagarto; la chinchilla, con la ardilla; el guacamayo, con el papagayo; y el manatí (manato), con el tiburón y el buey marino. Asimismo, el sistema de definición basado en la remisión de un animal a otro con el que comparte alguna característica también se aplica a los animales europeos (la ardilla, con la rata y la 'fuína' (comadreja); el tiburón, con el perro y el lobo (marino)).

14 En relación con las citas, la RAE afirma que "en unas [entradas] se ponen para autoridád, y en otras para exemplo [...]; pero aunque la Academia [...] ha elegido los Autóres que la han parecido haver tratado la Lengua con mayor gallardía y elegáncia, no por esta razón se dexan de citar otros, para comprobar la naturaleza de la voz [...]" (DA, Prólogo, V). Por su parte, algunas 'autoridades' del DA adolecen de cierta incoherencia: la cita remite en iguana al lagarto ("aunque su vista es bien asquerosa, pues parecen puros lagartos de España"), cosa que no sucede en lagarto ("Padecieron tanta hambre, que ni sapos, ni ranas, ni lagartos, ni otras cosas vivas, por sucias, dexavan de comer "). 
Tampoco es regular la RAE en su criterio ortográfico. Por mucho que reconozca que escribir cocodrilo es "en contra la practica de los mas selectos Autores y Vocabularios y contra su origen latino Crocodrilus" y que remita cocodrilo a crocodrilo, leemos "mui semejante al Cocodrilo" (s. v. caiman). Algo parecido sucede con la chinchilla, parangonada con la 'ardilla' (arda), voz escrita con hache ("Animaléjo pequeño como hardilla", s. v. chinchilla) a pesar de recomendar lo opuesto en arda ("Covarr. [...] le dá la etymología del verbo Arder por ser mui ardiente, fogosa e inquieta, por cuya razón se debe escribir esta voz sin h").

Lo anterior muestra, en primer lugar, que la RAE en el Diccionario de Autoridades heredó un modo de comprender América, al tiempo que se inscribió en la tradición lexicográfica precedente, en la que el diccionario y la enciclopedia permanecían estrechamente vinculados. En segundo lugar, que la falta de coherencia en el tratamiento lexicográfico de los indigenismos considerados está en consonancia con el enciclopedismo de las fuentes americanas del DA (las crónicas de Indias $)^{15}$-que las entradas pertenezcan a la zoología también favorece el enciclopedismo-; en tercer lugar, que no mencionar al animal americano en la parte definitoria del europeo responde al hecho de que este era conocido por el usuario y aquel no.

15 La huella de los textos cronísticos en el DA no es desdeñable en vista de que singulariza los diccionarios castellanos de los de otras lenguas. Así, en otros trabajos nos hemos ocupado de la transmisión de algunos indoamericanismos al catalán y al italiano (vid. Cala Carvajal 2000, 2001a y b), y hemos concluido que en la lexicografía de estas dos lenguas las notas enciclopédicas en la microestructura de préstamos léxicos indígenas son menores dado que aquella huella o ha desaparecido o nunca existió, con lo que sus entradas ganaron en precisión, concisión y claridad. 


\section{BIBLIOGRAFÍA}

AhUmada LARA, I. (1989): Aspectos de lexicografía práctica, Granada, Universidad de Granada.

Aliaga Jiménez, J. L. (1994): El léxico aragonés en el Diccionario de Autoridades (Real Academia Española), Zaragoza, Institución "Fernando El Católico".

Alvar EzquerRa, M. (1993a): "¿Qué es un diccionario? Al hilo de unas definiciones académicas”, en Lexicografía descriptiva, Barcelona, Biblograf, pp. 73-77.

_ (1993b): "La recepción de americanismos en los diccionarios generales de la lengua", en Lexicografía descriptiva, Barcelona, Biblograf, pp. 344-351.

— (1995): "Los diccionarios españoles en su historia", International Journal of Lexicography, VIII/3, pp. 174-201.

ANGLADA ARBoIX, E. (1991): "Lexicografía, metalexicografía, diccionario, discurso", Sintagma, 3, pp. 5-11.

AZORÍN FERNÁNDEZ, D. (2000): Los diccionarios del español en su perspectiva histórica, Alicante, Universidad de Alicante.

BORRERO BARRERA, M. J. (2000-2001): "Acerca de las personas gramaticales yo y nosotros frente al otro y al ellos en las crónicas de Indias: los Naufragios de Alvar Núñez Cabeza de Vaca", Humanística, 12, pp. 11-23.

— y R. CAla CARvajal (en prensa): "Diccionario y crónicas de Indias. La intertextualidad en la definición de indoamericanismos relativos a la fauna", en Actas del III Congreso Internacional de la Sociedad Española de Historiografía Lingüística (2001).

BosQue, I. (1982): "Sobre la teoría de la definición lexicográfica", Verba, 9 , pp. 105-123.

Buesa, T. y J. M. Enguita (1992): Léxico del español de América: su elemento patrimonial e indígena, Madrid, Mapfre.

BURIDANT, C. (1986): "Lexicographie et glossographie médievales. Esquisse de bilan et perspectives de recherche", en C. Buridant et alii, La lexicographie au Moyen Age, Lille, Presses Universitaires, pp. 9-46.

CALA CARVAJAL, R. (2000): "Las relaciones lingüísticas entre Cataluña y América. El caso de las voces caimán/caiman, carey/carei y pavo/gall dindi en la lexicografía castellana y catalana", Sintagma, 12, pp. 7193.

_ (2001a): "Las voces prehispanas 'cacao, cacahuete y maíz' en diccio- 
narios de lengua castellana, catalana e italiana del siglo XX", Boletín Americanista, 51, pp. 25-41.

- (2001b): "El español de América y el tipo del indiano en el teatro de Santiago Rusiñol", Hesperia. Anuario de Filología Hispánica, IV, pp. 15-30.

CASARES, J. (1992): Introducción a la lexicografía moderna, Madrid, CSIC [1950].

Clarinval, B. (1967): "Essai sur le statut linguistique du nom propre", Cahiers de Lexicologie, 11, pp. 29-44.

COTARElo y Mori, E. (1914): "La fundación de la Real Academia Española, y su primer director: don Juan Manuel Fernández Pacheco, marqués de Villena", BRAE, I, pp. 4-38 y 89-127.

DA: Real Academia Española (1984): Diccionario de Autoridades, Madrid, Gredos [1726-1739] (ed. facsímil).

DCCA: TERREROS Y PANDO, E. (1987): Diccionario castellano con las voces de ciencias y artes y sus correspondientes en las tres lenguas francesa latina e italiana, Madrid, Arco/Libros [1786-1793] (ed. facsímil).

DCEC: Corominas, J. y J. A. PASCuAl (1980): Diccionario crítico etimológico castellano e hispánico, Madrid, Gredos.

FERnÁndez Sevilla, J. (1974): Problemas de lexicografía actual, Bogotá, Instituto Caro y Cuervo.

Fontanella De Weinberg, M. B. (1992): El español de América, Madrid, Mapfre.

Frago Gracia. J. A. (1994): Andaluz y español de América: historia de un parentesco lingüístico, Sevilla, Junta de Andalucía.

FRAWLEY, W. (1981): "In defense of the Dictionary: A response to Haiman", Lingua, 55, pp. 53-61.

Galeote, M. (1997): Léxico indígena de flora y fauna (en Tratados de autores andaluces sobre las Indias Occidentales), Granada, Universidad de Granada.

Gili GAYA, S. (1963): La lexicografía académica del siglo XVIII, Oviedo, Universidad de Oviedo.

Guitarte, G. L. (1984): "La dimensión imperial del español en la obra de Aldrete: sobre la aparición del español en América en la lingüística hispánica”, Historiographia Linguistica, XI, 1/2, pp. 129-187.

Gútemberg, J. (1984): Concepto de 'americanismo' en la historia del español. Punto de vista lexicológico y lexicográfico, Bogotá, Instituto Caro y Cuervo. 
GutIÉRREZ CuADRADO, J. (1996): “Enciclopedia y diccionario", en E. Forgas (coord.), Léxico y diccionarios, Tarragona, Universitat Rovira i Virgili, pp. 133-159.

— y J. A. PASCUAL (1996): "El trabajo lingüístico de Jolis y la lexicografía novohispana del siglo XVIII", Lexis, XX, 1/2, pp. 333-371.

GutiérREZ RodiLLA, B. M. (1993): "Los términos relacionados con la medicina en el Diccionario de Autoridades", BRAE, LXXIII, pp. 463-512.

— - (1994-1995): "Construcción y fuentes utilizadas para los términos médicos en el Diccionario de Autoridades", Revista de Lexicografía, I, pp. 149-162.

— (1997-1998): "Plantas americanas con uso en medicina en el Diccionario de Terreros y Pando", Revista de Lexicografía, IV, pp. 107-124.

Haiman, J. (1980): “Dictionaries and Enciclopedias", Lingua, 50, pp. 329358.

—_ (1982): "Dictionaries and Enciclopedias Again", Lingua, 56, pp. 353355.

HAENSCH, G. (1999): Los diccionarios del español en el umbral del siglo $X X I$, Salamanca, Ediciones Universidad de Salamanca.

LARA, L. F. (1989): "Dictionnaire de langue, encyclopédie et dictionnaire encyclopédique: le sens de leur distinction", Wörterbücher. Dictionaries. Dictionnaires. Ein Internationale Handbuch zur Lexikographie, Berlin-New York, W. de Gruyter, vol. I, pp. 280-287.

LÁZARo CARRETER, F. (1980): "El primer diccionario de la Academia", Estudios de lingüística, Barcelona, Crítica, pp. 83-148.

Lecomte Hilmy, A. (1989): "Du statut linguistique des noms propes dans cinq dictionnaires français", Cahiers de Léxicologie, 54, pp. 8-32.

LOPE BLANCH, J. M. (1977): "Los indoamericanismos en el Tesoro de Covarrubias", NRFH, XXVI, pp. 296-315.

MARTINELl GifRE, E. (1992): La comunicación entre españoles e indios, Madrid, Mapfre.

MARTíNEZ DE SOUSA, J. (1995): Diccionario de lexicografía práctica, Barcelona, Biblograf.

Peréz Goyena, A. (1922): "Contribución de los jesuitas al Diccionario de Autoridades (1726-1739)", Razón y fe, 63, pp. 458-481.

POTTIER-NAVARRO, H. (1992): "El concepto de americanismo léxico", RFE, LXXII, pp. 297-312.

RABANALES, A. (1963): Introducción al estudio del español de Chile, Anexo $\mathrm{n}^{\circ}$ I del Boletín de Filología, Universidad de Chile. 
ReY-Debove, J. (1971): Étude linguistique et sémiotique des dictionnaires français contemporains, The Hague-Paris, Mouton.

RoMero, M. V. (1992): "Indigenismos en dos diccionarios españoles: Autoridades y Terreros", Las Indias (América) en la literatura del Siglo de Oro, Pamplona, Universidad de Navarra, pp. 265-83.

RonA, J. P. (1969): “¿Qué es un americanismo?”, Simposio de México, México, UNAM, pp. 135-149.

SAlVADOR RosA, A. (1985): "Las localizaciones geográficas en el Diccionario de autoridades", LEA, VII, pp. 103-139.

SECo, M. (1987a): "Problemas formales de la definición", en Estudios de lexicografía española, Madrid, Paraninfo, [1978], pp. 15-34.

_ (1987b): "El "contorno" en la definición", en Estudios de lexicografía española, Madrid, Paraninfo, [1979], pp. 35-45.

Sevilla, I. DE (1982): Etimologías, Madrid, Editorial Católica.

VAL, J. F. (1992): Las ideas gramaticales en el Diccionario de Autoridades, Madrid, Arco/Libros.

WeINREICH, U. (1970): "La definition lexicographique dans la sémantique descriptive", Langages, 19, pp. 69-86.

WERNER, R. (1982): "La definición lexicográfica", en G. Haensch et alii, La lexicografía. De la lingüística teórica a la lexicografía práctica, Madrid, Gredos, pp. 259-328.

\section{APÉNDICE}

\section{CORPus DE INDIGENISMOS ANALIZAdOS (DICCIONARIO DE AUTORIDADES)}

CAIMAN. Béstia Amphibia, mui semejante al Cocodrilo, de gran cuerpo y longítud, que se cría en las rias de las Indias y en algunas Islas. Es à modo de lagarto, con unas conchas tan fuertes y sólidas, que no las puede passar una bala de arcabúz. Tiene dos carreras de dientes, y debaxo de los brazos unas glándulas como habas, que despiden un olor suave, de que usan los Indios, los quales tienen gran destreza en cogerlos. Pone los huevos en la aréna, los que son tan duros, que no se pueden quebrar; pero se empollan con el calór del Sol. Es voz del País. Acost. Hist. Ind. Lib.3-cap.17. De los lagartos o caimánes que llaman, hai mucho escrito en histórias de Indias. ARGENS. Maluc. Lib.5.fol.165. En los rios y lagunas se crian caimánes horrendos y muchos.

IGUANA. s. m. Animal amphibio de la America, el qual es à manera de un lagarto, y de quien se trahen a España unas piedras llamadas de Iguana, que son prove- 
chosas para algunos males. Lat.Lacertus Americanus. ACOST. Hist.Ind.lib.4.cap.38. Harto mejor comida es la de Iguánas; aunque su vista es bien asquerosa, pues parecen puros lagartos de España.

CHINCHILLA. f. Animaléjo pequeño como hardilla, que se cria en la sierra del Perú. Tiene el pelo mui blando, y sus pieles se trahen por cosa regalada y saludable para abrigar el estómago, y partes que tienen necessidad de calór moderado. ACOST. Hist. Ind. Lib. 4. Cap. 38. Tambien se hacen cubiertas ó frazádas del pelo de estas chinchillas.

GUACAMAYO. s. m. Ave algo mayór que el papagáyo, y de su figúra. Tiene la pluma de varias y hermosas colores, y las de la cola mui largas. Son torpes para hablar, y jamás llegan à formar voces. Algunos los llaman Guacamáyas. Lat.Pseudopsithacus. HERR. Hist. Ind. Decad.7.lib.3.cap.13. Hai papagáyos, Guacamáyos, Pavas, Palomas torcazas, Tórtolas y Perdices de tres ó quatro manéras. INC. GARCIL. Coment.part.I.lib.8.cap.21. Son de muchas y diversas colóres, particularmente los grandes, que los Españoles llaman Guacamáyas.

MANATO. Especie de Tiburón, que se cria en el mar Indico, el qual tiene la boca como buey y el cuerpo mui gruesso, y del largo de veinte piés, cubierto de una piel duríssima è impenetrable. El lomo es llano, los ojos pequeños, y el colór pardillo. Tiene dos pies, que le salen como brazos de los hombros, de los quales se sirve para nadar. La hembra tiene dos grandes tetas, con cuya leche cria sus hijos, los quales pare vivos, como animal de tierra. Su carne, siendo fresca, tiene sabór de ternéra, y saláda tiene gusto de atún, aunque es mejór, y se conserva más tiempo. Su mantéca es mui buena, y nunca se enrancia, y con ella se adoba su mismo cuero, para hacer dél calzado y otras cosas. En la cabeza de este pescado dicen se cria una piedra mui provechosa contra las piedras de los riñones, y para los dolores de ijada. Lat. Vitulus marinus. HUERT. Plin. Lib.8. cap.31. Dicen algunos de los que navegan por el mar de España, que se hallan estos animales en él; pero mas ordinario es hallarlos en el mar Indico, donde tambien se cria el Manáto. 